\title{
CYP4A11 is involved in the development of nonalcoholic fatty liver disease via ROS-induced lipid peroxidation and inflammation
}

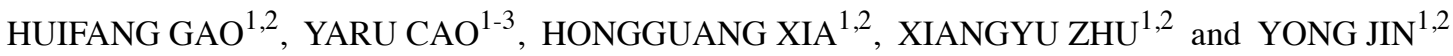

\author{
${ }^{1}$ Key Laboratory of Major Autoimmune Diseases, Anhui Institute of Innovative Drugs, School of Pharmacy, \\ Anhui Medical University; ${ }^{2}$ The Key Laboratory of Anti-inflammatory and Immune Medicines, Ministry of Education, \\ Hefei, Anhui 230032; ${ }^{3}$ Department of Pharmacy, Fourth People's Hospital of Wuhu City, Wuhu, Anhui 241002, P.R. China
}

Received June 20, 2019; Accepted December 16, 2019

DOI: $10.3892 / \mathrm{ijmm} .2020 .4479$

\begin{abstract}
Nonalcoholic fatty liver disease (NAFLD) is a fat metabolism disorder that occurs in liver cells. The development of NAFLD is considered to be associated with hepatic oxidative stress. The present study aimed to investigate the role of cytochrome P450 4A11 (CYP4A11) in the pathogenesis of NAFLD. The levels of plasma CYP4A11 and lipid peroxidation products levels exhibited a high correlation, and were increased significantly compared with those from normal subjects. Further in vitro studies demonstrated that the expression levels of CYP4A11 and the content of reactive oxygen species (ROS) were increased in free fatty acid (FFA)-stimulated HepG2 cells. Clofibrate, a CYP4A11 inducer, aggravated cell damage. Opposite results were observed for the CYP4A11 inhibitor HET0016, which attenuated apoptosis in FFA-treated cells. Furthermore, CYP4A11 gene overexpression and silencing were used to investigate the effects on inflammatory cytokine secretion. The data demonstrated that CYP4A11 promoted an increase in the mRNA expression of tumor necrosis factor $\alpha$, interleukin $(I L)-1 \beta$ and $I L-6$ in response to FFA. In addition, western blot analysis highlighted that CYP4A11 caused an upregulation of phosphorylated p65 levels and therefore affected the NF- $\mathrm{BB}$ signaling pathway. The data demonstrated that CYP4A11 may metabolize fatty acids to promote the production of ROS and accelerate the progression of NAFLD.
\end{abstract}

\section{Introduction}

Non-alcoholic fatty liver disease (NAFLD) is a progressive chronic liver disorder that is a global public health problem. The disease has exhibited increased prevalence due to the

Correspondence to: Professor Yong Jin, Key Laboratory of Major Autoimmune Diseases, Anhui Institute of Innovative Drugs, School of Pharmacy, Anhui Medical University, 81 Meishan Road, Hefei, Anhui 230032, P.R. China

E-mail: jinyong@ahmu.edu.cn

Key words: cytochrome P450 4A11, lipid peroxidation, nonalcoholic fatty liver disease, inflammatory, oxidative stress increasing rates of obesity and diabetes. NAFLD increases the risk of developing nonalcoholic steatohepatitis, liver fibrosis and liver cancer, due to the accelerated rates of inflammation and hepatocyte injury $(1,2)$. In China, sedentary lifestyles and excessive dietary fat intake are becoming the leading causes of obesity, metabolic syndrome and NAFLD (3). Oxidative stress has been proposed to be the primary mechanism of hepatocellular damage (4). Intracellular oxidative damage to cellular macromolecules is based on the disruption of the homeostasis between oxidative and antioxidative chemical species (5). This condition results from the release of toxic substances and inflammatory cytokines and contributes to the induction of hepatocyte apoptosis (4).

CYP4A is a member of the CYP4 enzyme family and is involved in the metabolism of medium- and long-chain fatty acids, such as arachidonic acid, palmitate, laurate and compounds with highly regionally selective hydroxylated terminal $\omega$-carbon (6). CYP4A is considered to serve a crucial role in the pathogenesis of NAFLD (7). CYP4A exhibits different pattern of metabolism across different species. Human CYP4A11 and CYP4A22, and mouse CYP4A10, CYP4A12 and CYP4A14 are responsible for the $\omega$-hydroxylation of fatty acids (8). CYP4A11 primarily metabolizes endobiotics and catalyzes the $\omega$-hydroxylation of fatty acids in human liver and kidney tissues. CYP4A11 is a functionally active member of the human CYP4A family of enzymes (9).

In patients with NAFLD, triglyceride (TG) synthesis was identified to be increased compared with that of the TG export from the liver cells (10). Liver free fatty acids (FFAs) that are derived from circulating non-esterified fatty acids (NEFAs) are stored as TGs. NEFAs are released from adipose tissues, hepatic de novo lipogenesis and the remnants of the TG rich lipoproteins, namely very-low-density lipoproteins (VLDL) and chylomicrons (11). These FFAs enter the mitochondria and undergo $\beta$-oxidation in order to produce ATP equivalents. Alternatively, they are esterified to triglycerides (TGs) and subsequently expelled from the hepatocytes as VLDLs (12). During the mitochondrial dysfunction caused by NAFLD, the CYP4-mediated $\omega$-hydroxylation of fatty acids is markedly increased (13). This process results in the excessive production of reactive oxygen species (ROS) (13). Cytochrome P450 (CYP) enzymes primarily depend on NADPH to produce superoxide and hydrogen peroxide (14). ROS serve a central 
role in activating $\mathrm{NF}-\kappa \mathrm{B}$. In addition, oxidative stress increases the release of pro-inflammatory cytokines and is associated with activation of the $N F-\kappa B$ signaling pathway $(15,16)$. Therefore, ROS production by CYP4A11 metabolism of fatty acids may cause inflammatory reactions.

In the present study, the CYP4A11 protein content was determined in the plasma of patients with NAFLD. In addition, the study aimed to investigate the regulation of oxidative stress and lipid peroxidation (LPO) by CYP4A11 in a cell line mode of NAFLD. These interactions were examined by inducing and inhibiting CYP4A expression in a cell line model, in order to affect the production of ROS.

\section{Materials and methods}

Patients and study design. In the present study, 59 patients with NAFLD and 30 normal healthy subjects were enrolled between March 2019 and April 2019. The study protocol was approved by The First Affiliated Hospital of the Anhui Medical University Institutional Review Board. Blood samples were collected at The First Affiliated Hospital of the Anhui Medical University following provision of informed consent for scientific research from the patients on March 4, 2019. The criteria for patient selection were as described previously (17): i) No history of alcohol consumption, or alcohol intake per week was $<140 \mathrm{~g}$ in men and $<70 \mathrm{~g}$ in women; ii) no presence of specific diseases that may result in fatty liver, such as viral hepatitis, drug-induced liver disease, total parenteral nutrition and Wilson's disease; iii) besides clinical manifestations of the primary disease, other non-specific symptoms and signs, such as fatigue, dyspepsia, dull liver pain and hepatosplenomegaly occur; iv) symptoms of metabolic syndromes, such as overweight and visceral obesity, hyperglycemia, blood lipid disorder and hypertension occur; v) mild to moderate increases in serum levels of transaminase and $\gamma$-glutamyl transpeptidase ( $<5$-fold the upper normal limit), usually presenting as an increase of alanine aminotransferase (ALT); vi) the results of the liver imaging studies met the imaging diagnostic criteria of diffuse fatty liver. In addition, laboratory tests were performed, including Color Doppler and blood biochemistry. The patients with liver disease selected did not suffer from kidney disease.

Biochemical analysis. After blood samples were centrifuged at $1,006 \mathrm{x} \mathrm{g}$ at $4^{\circ} \mathrm{C}$ for $15 \mathrm{~min}$, the serum concentrations of $\mathrm{TG}$, alanine aminotransferase (ALT) and aspartate aminotransferase (AST) were measured separately using TG (cat. no. A110-1-1), ALT (cat. no. C009-1-1), and AST commercial analysis kits (cat. no. C010-1-1; all Nanjing Jiancheng Bioengineering Institute Co., Ltd.).

Analysis of plasma levels of lipid peroxidation products (LPO) and of CYP4A11 expression. Laboratory investigations were performed using plasma samples. Following centrifugation at $1,341 \times \mathrm{g}$ at $4^{\circ} \mathrm{C}$ for $20 \mathrm{~min}$, the plasma supernatant was collected and stored at $-20^{\circ} \mathrm{C}$. LPO and CYP4A11 levels were quantified in plasma by the human LPO ELISA kit (cat. no. JL12392; Shanghai Jianglai Biological Technology Co., Ltd.) and the human CYP4A11 ELISA kit (cat. no. YX-032516H; Shanghai Preferred Biotechnology Co., Ltd.), respectively following a 5-fold dilution, according to the manufacturer's protocol.
FFA-induced steatosis. The liver cancer HepG2 cell line was purchased from Shanghai GeneChem Co., Ltd., and was seeded in a 6 -well plate. A total of $2.5 \times 10^{5}$ cells were plated and maintained in normal growth conditions for $24 \mathrm{~h}$. The cells were then treated with $1 \mathrm{mM}$ FFA for an additional $24 \mathrm{~h}$. The FFA solution used in the present study was a mixture of oleic acid (Sigma-Aldrich; Merck KGaA) and palmitic acid (Sigma-Aldrich; Merck KGaA) at a specific concentration ratio (2:1) as previously described (18). The expression of CYP4A11 was regulated by simultaneous treatment of the cells with $100 \mathrm{mM}$ clofibrate (Dalian Meilun Biotechnology Co., Ltd.) and FFA solution. HepG2 cells were incubated with $1 \mu \mathrm{M}$ HET0016 (Cayman Chemical Company) for $1 \mathrm{~h}$ at $37^{\circ} \mathrm{C}$ prior to the addition of FFA.

Oil Red $O$ staining. After incubation of HepG2 cells with FFA solution for $24 \mathrm{~h}$, the cells were washed gently with PBS and fixed with $10 \%$ neutral formaldehyde for $30 \mathrm{~min}$ at room temperature. The cells were then stained with freshly diluted Oil Red storage solution (3:2 ratio of Oil Red:deionized water) at room temperature for $10 \mathrm{~min}$. After the Oil Red $\mathrm{O}$ working solution was removed, the cells were washed with $60 \%$ isopropanol for 5 sec. Finally, the cells were observed under a fluorescent inverted microscope (Olympus Corporation).

Cell transfection. The small interfering RNA (siRNA) oligonucleotides against the CYP4A11 gene and the plasmids for CYP4A11 overexpression were both designed and synthesized by Shanghai Sangon Biotech Corporation. The siRNA sequences were as follows: Human CYP4A11-siRNA sense, 5'-CCGUGUGAGGAAUGCCUUUTT-3'; and antisense: 5'-AAAGGCAUUCCUCACACGGTT-3'; negative control (NC) sense: 5'-UUCUCCGAACGUGUCACGUTT-3'; and antisense: 5'-ACGUGACACGUUCGGAGAATT-3'. HepG2 cells were seeded at a density of $80-90 \%$ and were transfected with $20 \mu \mathrm{M}$ CYP4A11 small interfering RNA (siRNA) or $20 \mu \mathrm{M}$ CYP4A11 plasmid using Lipofectamine 2000 (Invitrogen; Thermo Fisher Scientific, Inc.) according to the manufacturer's protocol. Following $6 \mathrm{~h}$, the culture medium was changed and the FFA $(1 \mathrm{mM})$ solution was added to the cells for $24 \mathrm{~h}$.

Western blot analysis. HepG2 cells were lysed with RIPA lysis buffer (Beyotime Institute of Biotechnology) containing $1 \mathrm{mM}$ phenylmethylsulfonyl fluoride. The upper supernatant was collected following centrifugation at $4,024 \mathrm{x} \mathrm{g}$ at $4^{\circ} \mathrm{C}$ for $30 \mathrm{~min}$. The supernatant was quantitatively administered using a BCA protein kit. The protein (10 $\mu \mathrm{l} / \mathrm{lane})$ was separated by $10 \%$ SDS-PAGE. The proteins were electrotransferred to PVDF membranes (EMD Millipore). The membranes were washed in TBS-0.5\% Tween-20 buffer following blocking with $5 \%$ fat free milk powder for $2 \mathrm{~h}$ at room temperature and incubated with the specific primary antibodies at $4^{\circ} \mathrm{C}$ overnight. Horseradish peroxidase-conjugated goat anti-rabbit IgG or anti-mouse IgG antibodies(1:5,000, cat. nos. ZB-2301 or ZB-2305; Beijing Zhongshan Golden Bridge Biotechnology Co., Ltd.; OriGene Technologies, Inc.). were used for $1 \mathrm{~h}$ at room temperature for signal detection. The membrane was developed with enhanced chemiluminescence (Thermo Fisher Scientific, Inc.). Quantitative densitometric analyses of western blot gel images were performed using Image J 1.45s software 
Table I. Clinical and biochemical features of the patient cohort.

\begin{tabular}{lccc}
\hline Parameters & Control group & NAFLD group & P-value \\
\hline Sex (male/female) & $25 / 5$ & $44 / 15$ & - \\
Age, years (range, 24-91) & $54.57 \pm 17.30$ & $52.53 \pm 15.79$ & 0.58 \\
ALT, U/l (9-50) & $25.37 \pm 13.81$ & $30.56 \pm 16.47$ & 0.09 \\
AST, U/l $(15-40)^{\mathrm{a}}$ & $20.47 \pm 6.98$ & $22.76 \pm 6.38$ & $0.03^{\mathrm{b}}$ \\
$\mathrm{BMI}, \mathrm{kg} / \mathrm{m}^{2}$ & $21.43 \pm 1.83$ & $25.53 \pm 2.37$ & 0.06 \\
TG, mmol/l $(0.56-1.70)^{\mathrm{a}}$ & $1.52 \pm 1.69$ & $2.47 \pm 1.60$ & $0.04^{\mathrm{b}}$ \\
VLDL, mmol/l $(0.21-0.60)^{\mathrm{a}}$ & $0.56 \pm 0.26$ & $0.92 \pm 0.56$ & $0.04^{\mathrm{b}}$ \\
\hline
\end{tabular}

${ }^{a}$ The values in the brackets represent the normal range. ${ }^{\mathrm{b}} \mathrm{P}<0.05$ vs. control. The data are expressed as the mean \pm standard deviation. ALT, alanine transaminase; AST, aspartate transaminase; BMI, Body Mass Index; TG, triglyceride; VLDL, very-low-density lipoprotein cholesterol.

(National Institutes of Health). The following primary antibodies were used: CYP4A11 (1:1,000; cat. no. DF4749; Affinity Biosciences), p65 (1:500; cat. no. 21014; Signalway Antibody LLC), phosphorylated (p)-p65 (1:500, 11014; Signalway Antibody LLC), and $\beta$-actin (1:1,000; cat. no. TA-09; Beijing Zhongshan Golden Bridge Biotechnology Co., Ltd.; OriGene Technologies, Inc.).

Measurement of oxidative stress activity. ROS levels were measured using a ROS kit (Shanghai Beibo Biotechnology Co., Ltd.) according to the manufacturer's protocol. The fluorescent probe 2',7'-dichlorofluorescein (DCF) diacetate was added to HepG2 cells at $10 \mu \mathrm{M}$ and the cells were collected following incubation for $20 \mathrm{~min}$ at $37^{\circ} \mathrm{C}$ in the dark. The fluorescence intensity of DCF was excited at $488 \mathrm{~nm}$ and the emission was detected at $525 \mathrm{~nm}$ using an inverted fluorescence microscope (magnification x10). Fluorescence intensity was quantified using Image $\mathbf{J}$.

Measurement of malondialdehyde (MDA) and superoxide dismutase (SOD) levels. Following cell collection, the HepG2 cells were lysed and centrifuged at $4,024 \mathrm{x}$ g for $30 \mathrm{~min}$ at $4^{\circ} \mathrm{C}$ to obtain the supernatant samples. The levels of MDA were determined using an MDA assay kit (Beyotime Institute of Biotechnology) and SOD was measured using a SOD assay kit (Beyotime Institute of Biotechnology) according to the manufacturer's protocol. The protein concentration levels in the lysates were determined using a BCA protein assay kit (Beyotime Institute of Biotechnology). The results from the MDA and SOD assays are presented as nmol/mg and U/mg protein, respectively.

Determination of $m R N A$ levels using reverse transcription-quantitative polymerase chain reaction ( $R T-q P C R)$. Total RNA was extracted from cultured HepG2 cells using the TRIzol reagent (Invitrogen; Thermo Fisher Scientific, Inc.). The RNA was eluted with RNase-free water and the concentration was measured by UV detection at $260 \mathrm{~nm}$. Subsequently, cDNA was synthesized using the Transcriptor First-Strand cDNA Synthesis kit (Takara Bio, Inc.). qPCR was performed with SYBR-Green Master Mix (Takara Bio, Inc.). The thermocycling conditions were as follows: Initial denaturation at $95^{\circ} \mathrm{C}$ for $30 \mathrm{sec}$, followed by 40 cycles of $95^{\circ} \mathrm{C}$ for $10 \mathrm{sec}$ and $60^{\circ} \mathrm{C}$ for $30 \mathrm{sec}$. The RT-qPCR primers were purchased from Sangon Biotech Co., Ltd. The fold-change for mRNA relative to $\beta$-actin was determined using the $2^{-\Delta \Delta C q}$ method (19). The specific primer sequences were as follows: CYP4A11 (human) forward, 5'-ACGGCTTGCTCCTGTTGA ATGG-3'; CYP4A11 reverse, 5'-AGAGGTCAGGCTGTAGAT GGTGTC-3'; IL-6 (human) forward, 5'-CACACAGACAGC CACTCACC-3'; IL-6 reverse, 5'-AGTGCCTCTTTGCTG CTTTC-3'; TNF- $\alpha$ (human) forward, 5'-AACCTCCTCTCT GCCATCAA-3'; TNF- $\alpha$ reverse, 5'-CTGAGTCGGTCACCC TTCTC-3'; $I L-1 \beta$ forward, 5'-GGACAAGCTGAGGAAGAT GC-3'; $I L-1 \beta$ reverse, 5'-TCGTTATCCCATGTGTCGAA-3'; $\beta$-actin forward, 5'-TTGCTGACAGGATGCAGAA-3'; and $\beta$-actin reverse, 5'-ACCAATCCACACAGAGTACTT-3'.

Statistical analysis. The data are expressed as the mean \pm standard deviation. Pearson correlation analysis was used to analyze correlation between variables. A Student's t-test, or one-way analysis of variance followed by Tukey post hoc test were used to compare the differences among the various groups. $\mathrm{P}<0.05$ was considered to indicate a statistically significant difference. All statistical analyses were performed with the SPSS v.16.0 software (SPSS, Inc.).

\section{Results}

Detection of LPO and CYP4A11 levels in plasma samples. The clinical and biochemical characteristics of the patients with NAFLD and of the control subjects involved in the present study are presented in Table I. A total of $77.5 \%$ of the participants were male, with an age range of 24-91 years. No significant differences were noted in the ALT levels between the patients with NAFLD and the control subjects, whereas the AST levels were slightly increased in the patients with NAFLD. However, this increase was not significant, and the levels were within the normal range of reference values. In contrast to these results, the mean BMI value of the patients with NAFLD was significantly increased compared with that noted for the healthy subjects, and this value exceeded the threshold of obesity according to the body-mass index for Asian populations (20). Plasma VLDL and TG levels in the patients with NAFLD were significantly increased compared with those of the control subjects. 
A

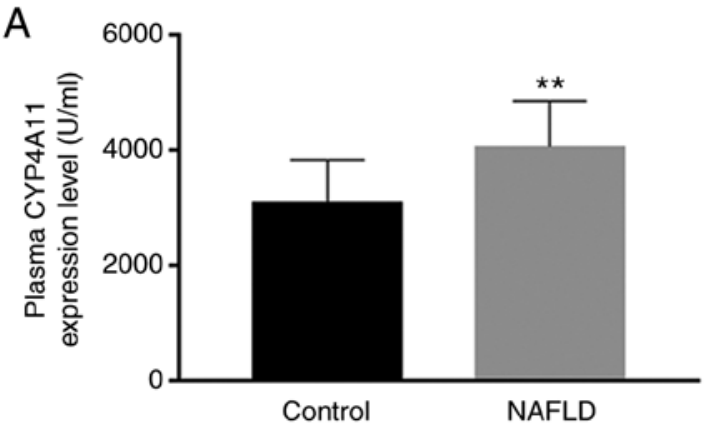

B

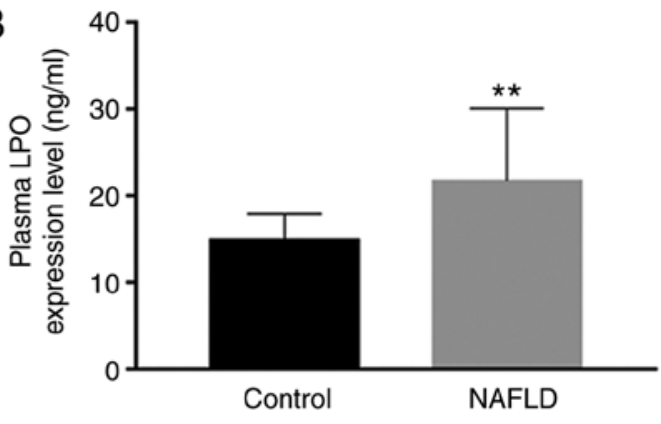

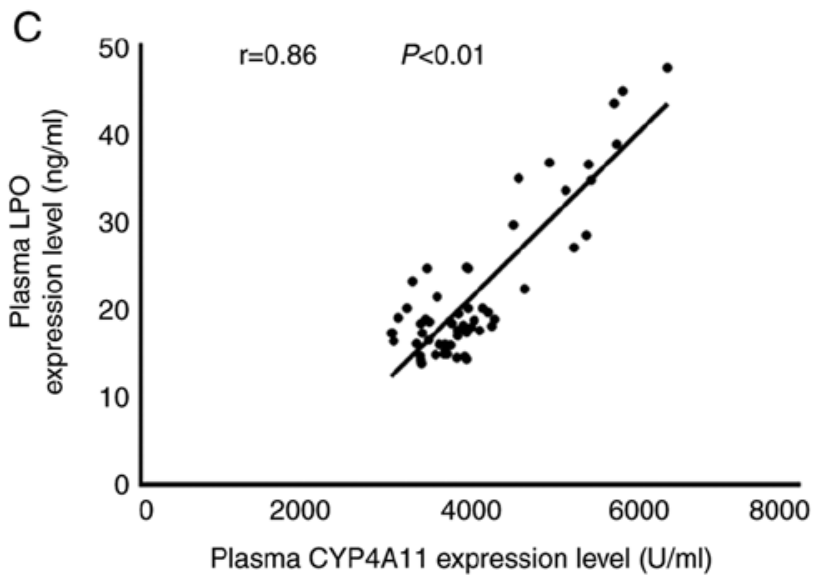

Figure 1. Linear association between plasma CYP4A11 and LPO levels in patients with NAFLD. Plasma samples were used to compare the changes in CYP4A11 and LPO between the patients with NAFLD and healthy controls. (A and B) Plasma (A) CYP4A11 and (B) LPO levels were determined by ELISA. (C) Correlation plots with linear regression comparing changes in the levels of CYP4A11 and LPO. The results are presented as the mean \pm standard deviation. ${ }^{* *} \mathrm{P}<0.01$ vs. control. CYP4A11, cytochrome P450 4A11; LPO, lipid peroxidation products; NAFLD, nonalcoholic fatty liver disease.

Plasma LPO levels were apparently elevated in the patients with NAFLD compared with those observed in the control subjects (Fig. 1). The plasma levels of CYP4A11 in the patients with NAFLD were significantly increased compared with those noted in control subjects. The levels of LPO were increased in a similar trend as that observed in the CYP4A11 levels in the patients with NAFLD. Further linear regression mapping indicated that these two parameters were closely associated $(\mathrm{r}=0.86)$.

Lipid accumulation in FFA-treated HepG2 cells. The effects of FFA treatment on lipid accumulation were confirmed by Oil Red O staining (Fig. 2A). The results indicated that the intracellular lipid deposition in the FFA-treated group was considerably increased compared with that of the control group. However, following the addition of HET0016 to the FFA-treated HepG2 cells, the level of lipid deposition was significantly decreased. The highest degree of lipid deposition was observed in the presence of clofibrate + FFA solution. The levels of the intracellular TGs followed the same trend (Fig. 2B).

Effect of FFA on the transaminase levels of HepG2 cells. Following FFA stimulation of the cells for $24 \mathrm{~h}$, the cell supernatant was collected for ALT and AST determination and the results are shown in Fig. $2 \mathrm{C}$. The results demonstrated that the levels of ALT and AST in the supernatant were increased in the FFA-treated HepG2 cells compared with those noted in the normal control group. HET0016 treatment decreased FFA-induced ALT and AST activity, whereas clofibrate promoted the FFA-induced activity of ALT and AST.
Detection of CYP4A11 expression in FFA-treated HepG2 cells. CYP4A11 levels were detected following FFA treatment of HepG2 cells. The protein and mRNA expression levels of CYP4A11 were elevated in FFA-treated HepG 2 cells compared with those of the normal control group (Fig. 2D and E). In addition, western blot analysis and RT-qPCR analysis demonstrated that clofibrate treatment resulted in a significant upregulation in the expression level of CYP4A11. In addition, FFA-induced CYP4A11 expression in HepG2 cells was inhibited by HET0016 treatment.

Association between CYP4A11 and oxidative stress. Pre-treatment of the HepG2 cells with FFA for $24 \mathrm{~h}$ increased the intracellular ROS content compared with that observed in the control group. In addition, the levels of ROS were significantly increased in the clofibrate group, as demonstrated by fluorescence detection. Conversely, HET0016 significantly inhibited the intracellular ROS production compared with that of the clofibrate group (Fig. 3A and B). The changes noted in the MDA levels were in accordance with the trend noted for the ROS levels among the different treatment groups. In contrast to the ROS and MDA levels, SOD levels were decreased following an increase in ROS levels (Fig. 3C and D).

Effects of CYP4A11 on inflammatory cytokine production in FFA-treated HepG2 cells. The expression levels of CYP4A11 were elevated following the transfection of pcDNA3.1-CYP4A11 into the HepG2 cells, as determined by western blot analysis and RT-qPCR. The levels of CYP4A11 in 
A

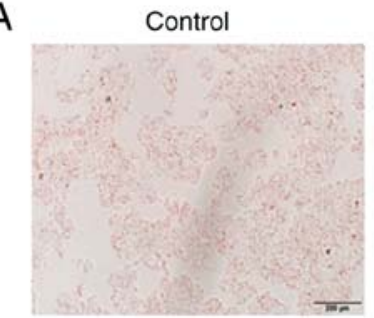

$\mathrm{FFA}+$ Clofibrate

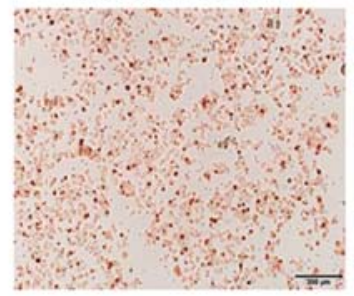

C

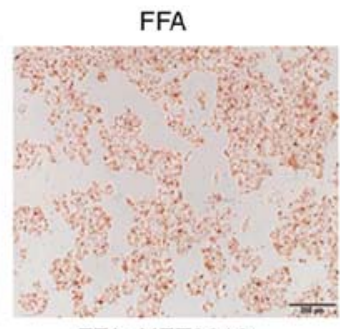

$\mathrm{FFA}+\mathrm{HET} 0016$

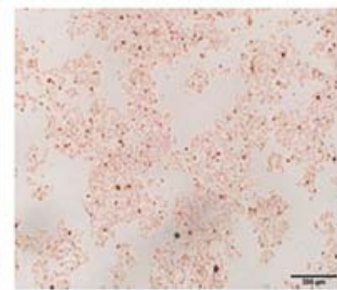

$\square$ Control

$\square$ FFA

FFA+HET0016

- FFA+Clofibrate

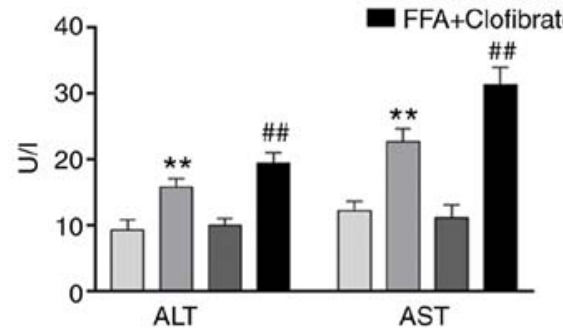

E

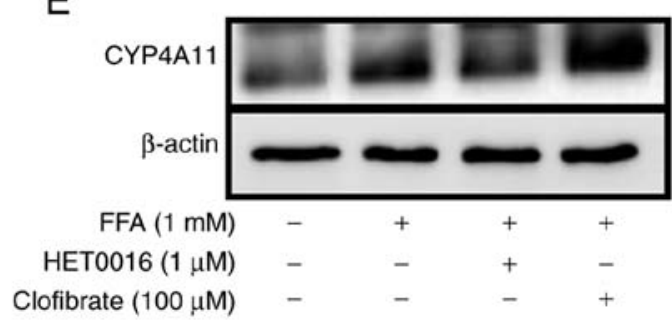

B

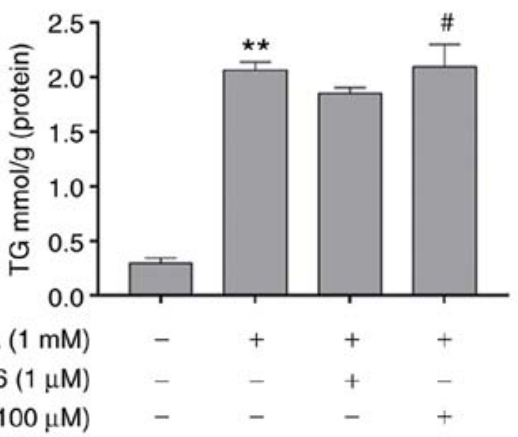

Clofibrate $(100 \mu \mathrm{M})$

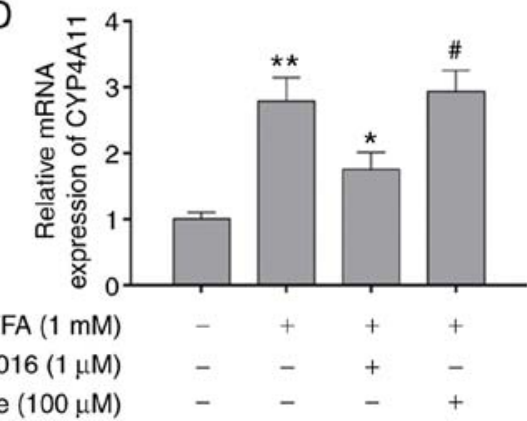

Clofibrate $(100 \mu \mathrm{M}) \quad-\quad-\quad-\quad+$

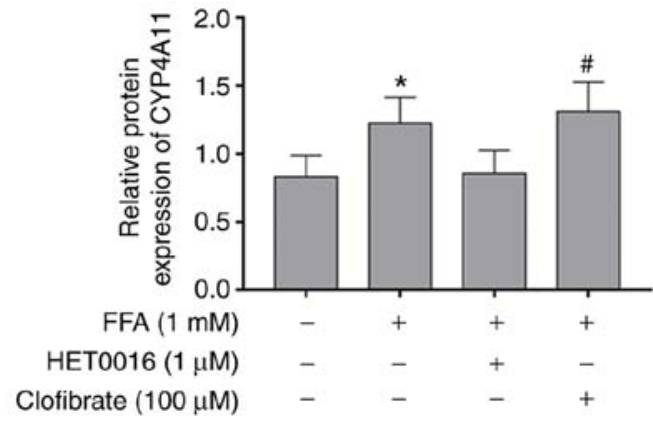

Figure 2. Effects of FFA, clofibrate and HET0016 on Oil Red staining, TG and CYP4A11 synthesis in HepG2 cells. HepG2 cells were treated with FFA (1 mM), FFA + clofibrate $(100 \mu \mathrm{M})$ or FFA + HET0016 $(1 \mu \mathrm{M})$ solution for $24 \mathrm{~h}$. (A) Lipid droplets in HepG2 cells were stained with Oil Red O. Scale bar, $200 \mu \mathrm{m}$. (B) The effects of these treatments on TG content in HepG2 cells was measured. (C) ALT and AST level in FFA-induced HepG2 cells. (D) The mRNA level of CYP4A11 was examined by reverse transcription-quantitative polymerase chain reaction. (E) The protein level of CYP4A11 was assessed by western blot analysis. The results are presented as the mean \pm standard deviation. ${ }^{*} \mathrm{P}<0.05$ and ${ }^{* *} \mathrm{P}<0.01$ vs. control. ${ }^{\#} \mathrm{P}<0.05$ and ${ }^{\# \#} \mathrm{P}<0.01$ vs. HET0016 group. FFA, free fatty acids; TG, triglyceride; CYP4A11, cytochrome P450 4A11; ALT, alanine aminotransferase; AST, aspartate aminotransferase.

the pCDNA3.1-CYP4A11 group were significantly increased compared with those of the model group (Fig. 4A and B). Moreover, the expression levels of the inflammatory cytokines TNF- $\alpha$, IL- 6 and IL- $1 \beta$ were examined by RT-qPCR. The expression levels of TNF- $\alpha$, IL- 6 and IL-1 $\beta$ were significantly increased in the pCDNA3.1-CYP4A11 group (Fig. 4E). The role of CYP4A11 on the secretion of inflammatory cytokines was further confirmed by transfection of siRNA-CYP4A11. The results demonstrated that the mRNA and protein expression levels of CYP4A11 in the siRNA-CYP4A11 group were significantly decreased (Fig. 4C and D). With regard to inflammatory cytokine secretion, RT-qPCR analysis demonstrated that $C Y P 4 A 11$ gene silencing inhibited the expression levels of $T N F-\alpha, I L-6$ and $I L-1 \beta$ (Fig. $4 \mathrm{~F}$ ).

Effect of CYP4A11 on the $N F-\kappa B$ signaling pathway in vitro. The expression levels of the primary protein associated with the $N F-\kappa B$ signaling pathway were detected by western blot analysis. The levels of p-p65 were apparently increased following the overexpression of CYP4A11 compared with those of the vector group (Fig. 4B). CYP4A11-siRNA inhibited the expression of p-p65 compared with that of the siRNA-NC group (Fig. 4D).

\section{Discussion}

It has been demonstrated that CYP4A activity is mediated via peroxisome proliferator-activated receptor $\alpha(\operatorname{PPAR} \alpha)$ activation and that it is involved in hepatic fatty acid disposal, leading to an increase in the levels of ROS and LPO (21). A previous study considered CYP4A as a potential target for the treatment of fatty liver disease (22). CYP4A induces oxidative stress by enhancing NADPH-dependent superoxide anion generation. The primary sources of ROS production 
A

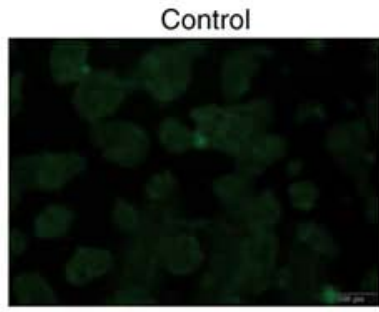

FFA+HET0016

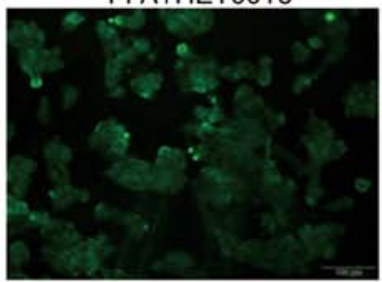

C

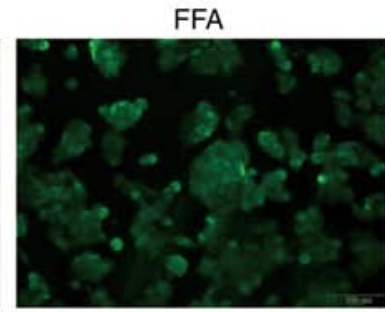

FFA+Clofibrate

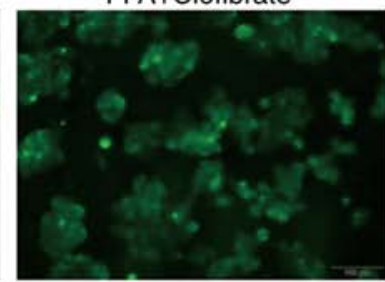

.

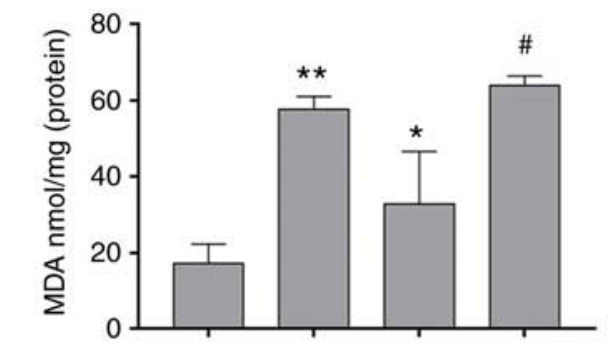

FFA (1 mM)

HET0016 $(1 \mu \mathrm{M})$

Clofibrate $(100 \mu \mathrm{M})$

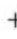

$-$

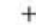

$+$
$+$

$-$
B

FFA $(1 \mathrm{mM})$

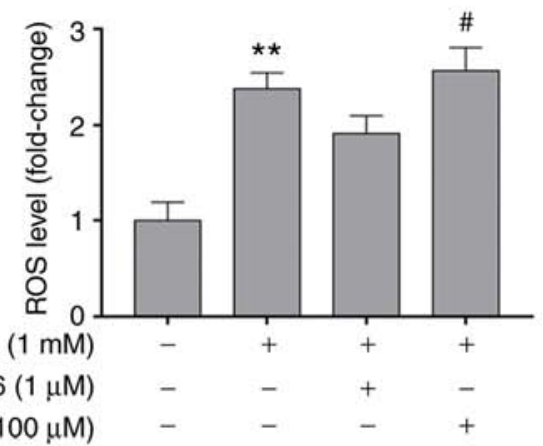

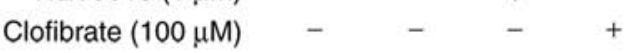

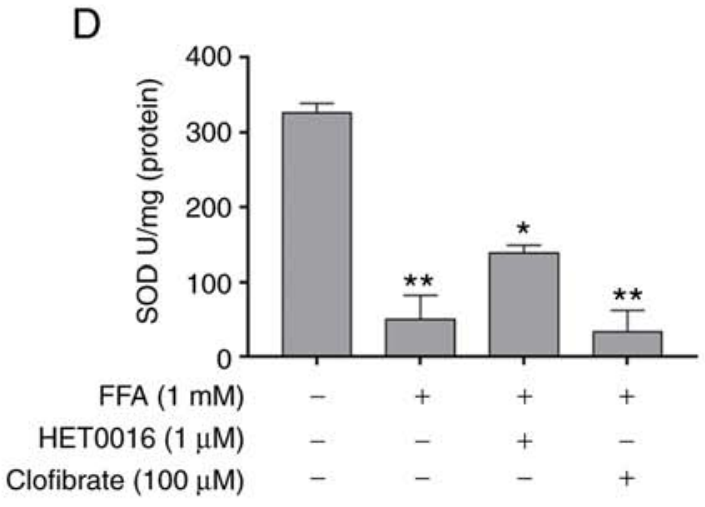

Figure 3. Effects of FFA, clofibrate and HET0016 on the synthesis of ROS, MDA and SOD in HepG2 cells. FFA induced hepatic oxidative stress and lipid peroxidation in HepG2 cells, and the effects of clofibrate and HET0016 on FFA-induced HepG2 cells were measured. (A) ROS was analyzed by fluorescence. Images are at magnification x10. Scale bar, $100 \mu \mathrm{m}$. (B) Quantification of ROS content. (C and D) Effects of changes in the levels of (C) MDA and (D) SOD in the experimental groups. The results are presented as the mean \pm standard deviation. ${ }^{*} \mathrm{P}<0.05$ and ${ }^{* * *} \mathrm{P}<0.01$ vs. control. ${ }^{\#} \mathrm{P}<0.05$ vs. HET0016 group. FFA, free fatty acids; ROS, reactive oxygen species; MDA, malondialdehyde; SOD, superoxide dismutase.

that can cause oxidative stress damage to the cells are $\mathrm{H}_{2} \mathrm{O}_{2}$, singlet oxygen and lipid hydroperoxides $(23,24)$. Excessive levels of ROS are the result of a disruption of the homeostasis between ROS production and the antioxidant system (25). Mitochondrial CYP2E1 is a direct source of ROS and it has also been demonstrated to have a greater ability to generate ROS compared to CYP3A4 and CYP1A1 $(26,27)$. CYP2E1 and CYP4A are vital hepatic microsomal oxidases associated with fatty acid oxidation and the formation of ROS and NOS (24). In addition, similar to CYP2E1, CYP4A is an alternative initiator of oxidative stress and promotes LPO in non-alcoholic steatohepatitis (NASH) (22). Although CYP4A serves an important role in the regulation of oxidative stress and LPO during a high-fat diet, it is not clear whether it promotes the development of NAFLD. CYP4A11 is considered to be an active $\omega$-hydroxylase in the human liver and is a major member of the human CYP4A family of enzymes. Another member of the human CYP4A family is the enzyme CYP4A22, which is not considered to serve a major role in the metabolism of fatty acids, as its expression level is extremely low in the human liver (28). In the present study, the functional role of CYP4A11 was primarily investigated.

It was initially demonstrated that the expression levels of CYP4A11 were increased in the plasma of patients with NAFLD, as determined by ELISA. Although this method did not directly determine the amount of protein per liver tissue, it was considered to be a rough estimation of the changes that occurred in the expression of CYP4A11 in the liver of patients with NAFLD. This is due to the limited applications of liver biopsy methods, which are not commonly used to detect the presence of NAFLD. Therefore, it is particularly difficult to obtain liver tissue. Plasma LPO levels were measured by ELISA. The present study sought to identify a potential association between CYP4A11 expression and LPO levels in the plasma of patients with NAFLD. CYP4A is associated with hepatic microsomal LPO and previous studies have reported that this enzyme may be responsible for the increase of in vitro microsomal LPO $(14,21)$. The present data indicated that hepatic CYP4A11 was significantly increased and that the plasma LPO levels were upregulated in patients with NAFLD. The linear regression between CYP4A11 expression and LPO levels indicated that they were highly correlated. The present study suggested that CYP4A11 was involved in LPO in patients with NAFLD.

The majority of the aforementioned studies involved animal experiments. In order to further understand the mechanism of the association between CYP4A11 expression and LPO levels in vitro, cell-based assays were conducted in the present study. An in vitro NAFLD model was established by pre-treating HepG2 cells with FFAs, which was similar to certain previ- 

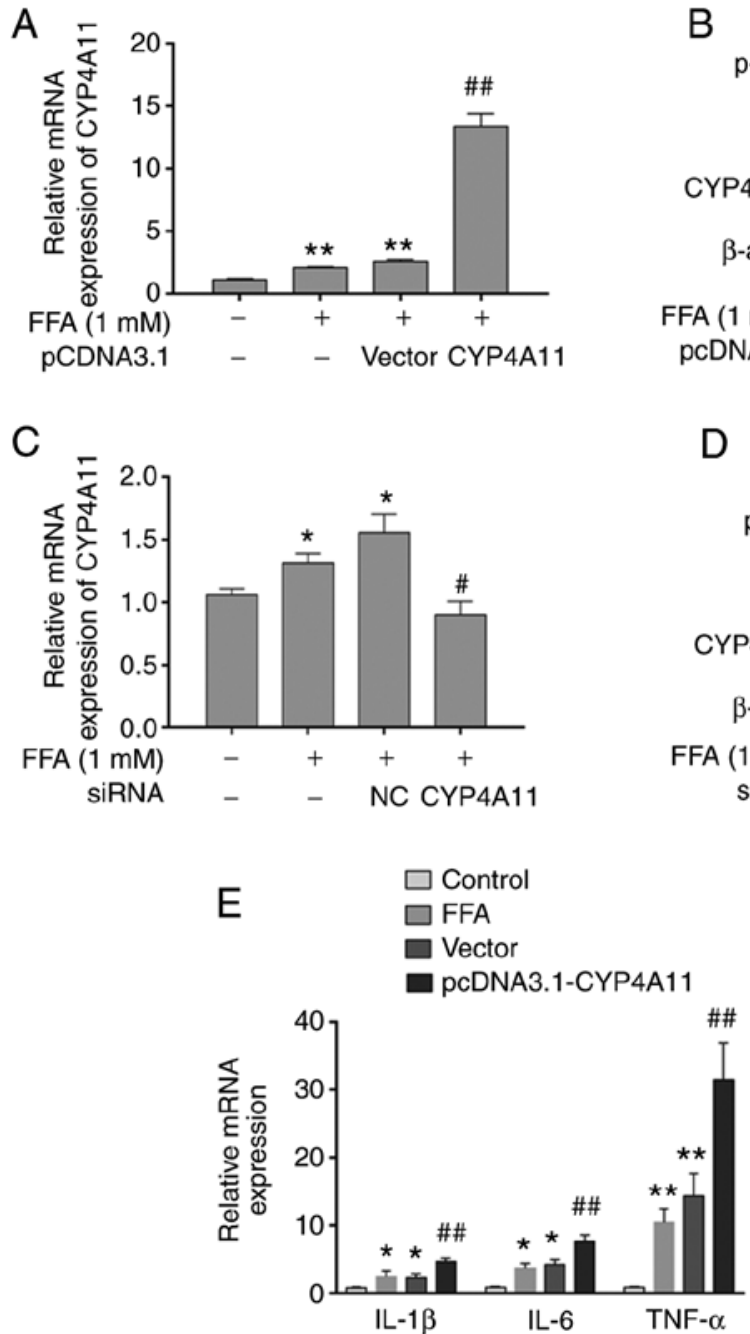
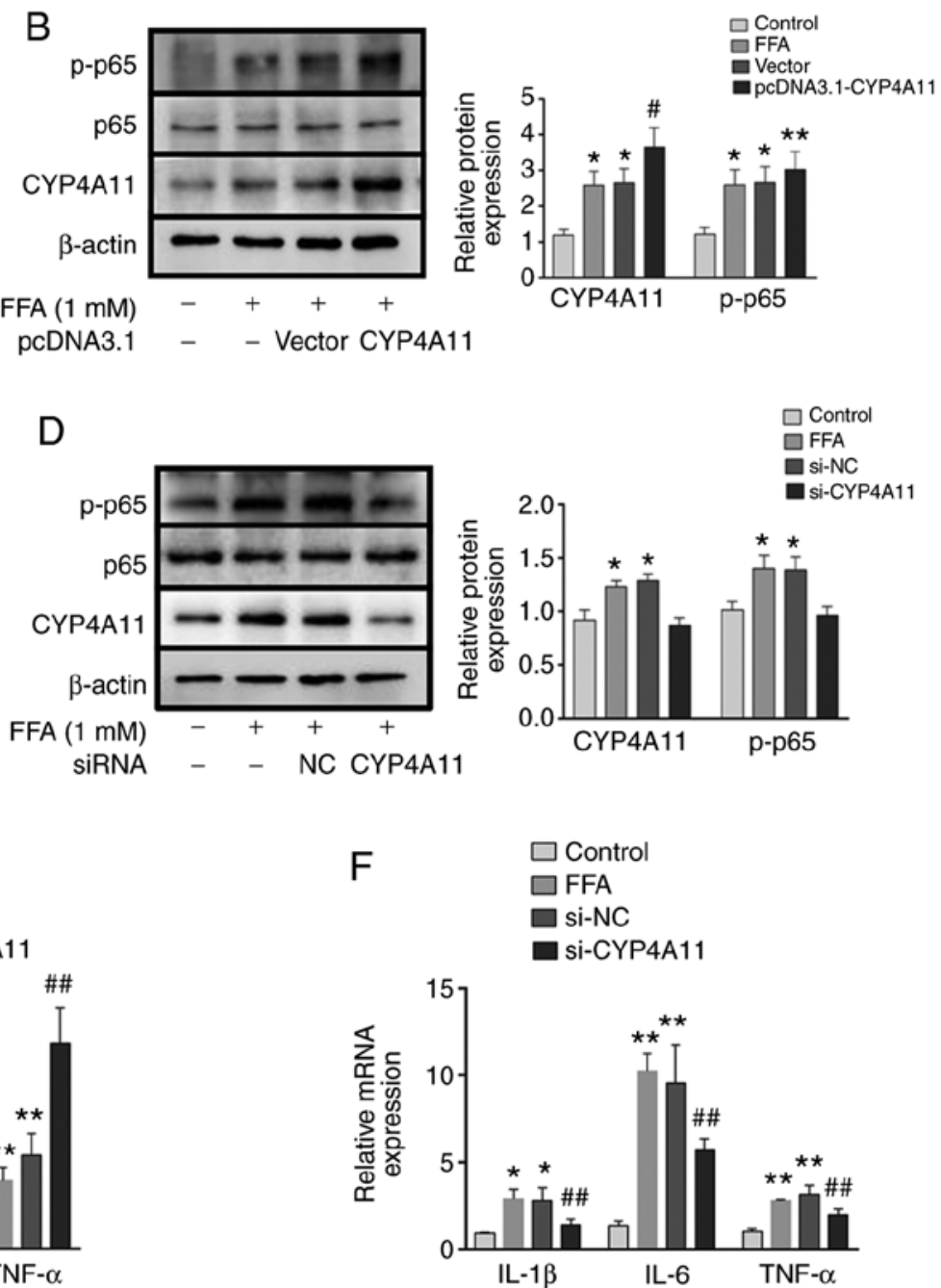

Figure 4. Effect of CYP4A11 gene on phosphorylation of p65 protein and synthesis of TNF- $\alpha$, IL-6 and IL-1 $\beta$. (A and C) The mRNA level of CYP4A11 was examined by RT-qPCR in the (A) pCDNA3.1-transfected and (C) siRNA-transfected groups. (B and D) The protein levels of CYP4A11 and p-p65 in (B) pCDNA3.1-transfected and (D) siRNA-transfected groups were assessed by western blot analysis. (E and F) IL-1 $\beta$, IL-6 and TNF- $\alpha$ expression levels were determined by RT-qPCR in the (E) pCDNA3.1-transfected and (F) siRNA-transfected groups. The results are presented as the mean \pm standard deviation ${ }^{*} \mathrm{P}<0.05$ and $^{* *} \mathrm{P}<0.01$ vs. control, ${ }^{\#} \mathrm{P}<0.05$ and ${ }^{\# \#} \mathrm{P}<0.01$ vs. vector or NC group. CYP4A11, cytochrome P450 4A11; p65, transcription factor p65; TNF- $\alpha$, tumor necrosis factor $\alpha$; IL, interleukin; RT-qPCR, reverse transcription-quantitative polymerase chain reaction; siRNA, small interfering RNA; p-, phosphorylated; $\mathrm{NC}$, negative control.

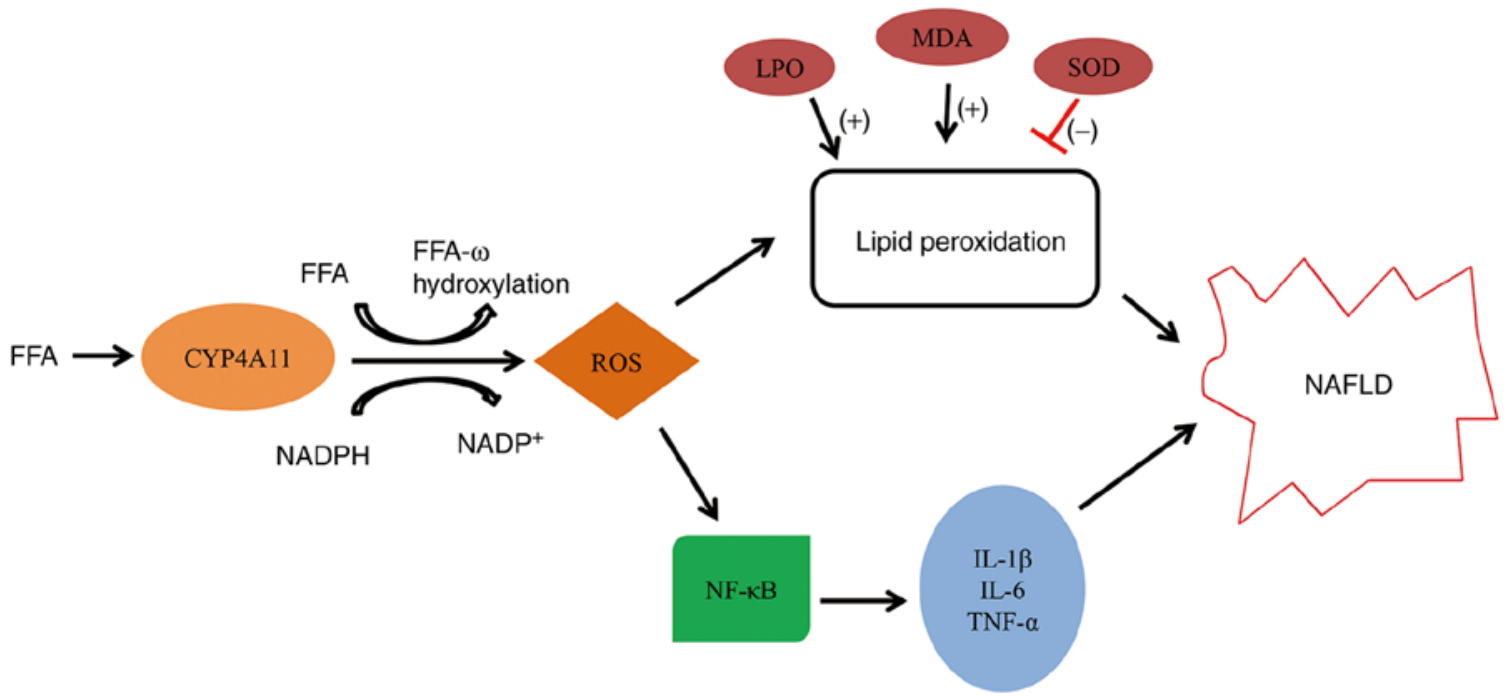

Figure 5. A potential pathway of NAFLD development. FFA, free fatty acids; CYP4A11, cytochrome P450 4A11; ROS, reactive oxygen species; LOPs, lipid peroxidation products; MDA, malondialdehyde; SOD, superoxide dismutase; IL, interleukin; TNF- $\alpha$, tumor necrosis factor $\alpha$; NAFLD, nonalcoholic fatty liver disease. 
ously described in vivo NAFLD models (29). FFA is a suitable stimulus for HepG2 cells as it is more steatogenic and causes less damage compared with PA, thereby increasing intracellular TG accumulation (18). In the present study, the results demonstrated that the expression levels of CYP4A11 in HepG2 cells treated with FFA were significantly increased, corresponding to the high CYP4A expression induced by high-fat diet or methionine and choline-deficient diet in animal models $(30,31)$. This is attributed to a large amount of FFA entering the cell that can maximize $\beta$-oxidation in the mitochondria, leading to the activation of the peroxisome $\beta$-oxidation and the microsomal $\omega$-oxidation pathways, which in turn increases CYP4A11 expression (32). Clofibrate, a PPAR $\alpha$ agonist, increases the expression of CYP4A (33). The addition of clofibrate to FFA will further increase the expression of CYP4A11. HET0016 is a known CYP4 family inhibitor that selectively inhibits the formation of 20-HETE (34). In the present study, the highest expression level of CYP4A11 protein was observed following addition of clofibrate, whereas HET0016 treatment decreased the expression levels of CYP4A11 to approximately the same levels as those noted for the normal group. CYP4A enzymes have been demonstrated to induce liver fat accumulation by increasing the expression of platelet glycoprotein 4 (22). The present study demonstrated that the increase in the expression levels of CYP4A11 increased the synthesis of TG, while the inhibition of CYP4A11 expression decreased the synthesis of TG. Therefore, CYP4A11 was closely associated with the production of TG, affecting the lipid toxicity of HepG2 cells and the increase in the levels of ALT and AST.

Elevated plasma FFA concentration leads to high intracellular ROS production due to the metabolism of FFAs in the mitochondria, peroxisomes and microsomes, which are considered the primary sources of ROS that cause oxidative stress $(15,35)$. The present study demonstrated that clofibrate-induced CYP4A11 expression may increase ROS levels by increasing FFA, while HET0016 attenuated ROS production by inhibiting CYP4A11 expression. These data indicated that CYP4A11 may promote the production of ROS. ROS induces LPO of membranes leading to cell necrosis or apoptosis. If ROS scavengers are used, we hypothesize that ROS scavengers will greatly decrease cytotoxic damage, but they may have little effect on the CYP4A11 effect. MDA is released as a result of LPO, which triggers a harmful immunological response to liver cells (36). In the present study, the severity of the antioxidant system disorders was reflected by the decrease of SOD concentration and the increased deposition of MDA, which were closely associated with the increase in ROS levels.

FFAs may be considered important mediators of lipotoxicity due to their potential cytotoxicity. In addition, they can lead to excessive accumulation of lipids and further stimulate TNF- $\alpha$ expression (37). ROS production and the products of LPO have been identified as a trigger for the production of proinflammatory cytokines, which in turn leads to neutrophil chemotaxis and development of diverse lesions in NASH subjects (38). The present study demonstrated that the mRNA expression levels of $T N F-\alpha, I L-6$ and $I L-1 \beta$ were significantly increased in the FFA-treated HepG2 cells. The expression levels of CYP4A11 were controlled at the genetic level, in order to cause an amplification of its effect on the expression of pro-inflammatory cytokines. The expression levels of TNF- $\alpha$, IL- 6 and IL-1 $\beta$ were increased following pCDNA3.1-CYP4A11 transfection into the cells. In contrast to this model, knockdown of CYP4A11 was achieved by siRNA transfection. The mechanism by which CYP4A11 regulates the expression of pro-inflammatory cytokines may be due to ROS and lipid peroxide production induced by CYP4A11. Excess ROS production and release of pro-inflammatory cytokines can activate NF-Kb $(39,40)$. ROS levels can activate and inhibit $\mathrm{NF}-\kappa \mathrm{B}$ signaling. The interaction between ROS and the NF- $\mathrm{KB}$ pathway is complex (41). The present study further investigated whether ROS induction by CYP4A11 affected the activation of the NF- $\mathrm{KB}$ signaling pathway. The data confirmed that upregulation of CYP4A11 by pCDNA3.1-CYP4A11 increased the expression levels of the NF- $\mathrm{KB}$ signaling pathway-associated protein p-p65. In addition, a reverse trend was noted in the presence of si-CYP4A11. This result suggested that CYP4A11 may affect the activation of the NF- $\kappa$ B signaling pathway, which may be mediated by ROS production (Fig. 5).

Taken collectively, the data of the present study demonstrated the significant role of CYP4A11 in the metabolism of fatty acids. CYP4A11 may serve as a mediator of oxidative stress and LPO. However, additional experimental studies are required to fully investigate the mechanisms of fatty acid metabolism by CYP4A11, in order to aid the development of novel therapeutic strategies for the treatment of NAFLD.

\section{Acknowledgements}

The authors would like to thank Mr Yuan Hong Xu of the First Affiliated Hospital of Anhui Medical University for assisting with the collection of patient blood samples.

\section{Funding}

The present study was supported by the Anhui Province Natural Science Foundation (grant no. 1808085MH235) and the Scientific Research Foundation of the Institute of Anhui Province Transformational Medicine (grant no. 2017zhyx32).

\section{Availability of data and materials}

The datasets used and/or analyzed during the current study are available from the corresponding author on reasonable request.

\section{Authors' contributions}

HG and YJ conceived the study and analyzed the relevant literature. YC, HX and XZ performed the literature review and were involved in conducting experiments. HG and YJ performed experiments, and were involved in writing, reviewing and editing the manuscript. All authors read and approved the final version of the manuscript.

\section{Ethics approval and consent to participate}

All experiments were authorized by the Biomedical Ethics Committee of Anhui Medical University. All procedures involving human participants were performed in accordance with the ethical standards of the institutional, and with the 1964 Helsinki declaration and its later amendments or comparable 
ethical standards. Written informed consent was provided by each patient.

\section{Patient consent for publication}

Written informed consent was provided by each patient.

\section{Competing interests}

The authors declare that they have no competing interests.

\section{References}

1. Bedossa P: Pathology of non-alcoholic fatty liver disease. Liver Int 37: 85-89, 2017.

2. Brunt EM: Pathology of nonalcoholic fatty liver disease. Nat Rev Gastroenterol Hepatol 7: 195-203, 2010.

3. Loomba R and Sanyal AJ: The global NAFLD epidemic. Nat Rev Gastroenterol Hepatol 10: 686-690, 2013.

4. Basaranoglu M, Basaranoglu G and Senturk H: From fatty liver to fibrosis: A tale of 'second hit'. World J Gastroenterol 19 1158-1165, 2013.

5. Robertson G, Leclercq I and Farrel GC: Nonalcoholic steatosis and steatohepatitis II. Cytochrome P-450 enzymes and oxidative stress. Am J Physiol Gastrointest Liver Physiol 281: G1135-G1139, 2001.

6. Sharma RK, Doig MV, Lewis DF and Gibso GG: Role of hepatic and renal cytochrome P-450 IVA1 in the metabolism of lipid substrates. Biochem Pharmacol 38: 3621-3629, 1989.

7. Leclercq IA, Farrell GC, Field J, Bell DR, Gonzalez FJ and Robertson GR: CYP2E1 and CYP4A as microsomal catalysts of lipid peroxides in murine nonalcoholic steatohepatitis. J Clin Invest 105: 1067-1075, 2000

8. Roman RJ: P-450 metabolites of arachidonic acid in the control of cardiovascular function. Physiol Rev 82: 131-185, 2002.

9. Kim D, Cha GS, Nagy LD, Yun CH and Guengerich FP: Kinetic analysis of lauric acid hydroxylation by human cytochrome P450 4A11. Biochemistry 53: 6161-6172, 2014.

10. Zhang J, Hu J, Zhang C, Jiao Y, Kong X and Wang W: Analyses of risk factors for polycystic ovary syndrome complicated with non-alcoholic fatty liver disease. Exp Ther Med 15: 4259-4264, 2018.

11. Shojaee-Moradie F, Cuthbertson DJ, Barrett M, Jackson NC, Herring R, Thomas EL, Bell J Kemp GJ, Wright $\mathbf{J}$ and Umpleby AM: Exercise training reduces liver fat and increases rates of VLDL clearance but not VLDL production in NAFLD. J Clin Endocrinol Metab 101: 4219-4228, 2016.

12. Mansouri A, Gattolliat $\mathrm{CH}$ and Asselah T: Mitochondrial dysfunction and signaling in chronic liver diseases. Gastroenterology 155: 629-647, 2018.

13. Hardwick JP: Cytochrome P450 omega hydroxylase (CYP4) function in fatty acid metabolism and metabolic diseases. Biochem Pharmacol 75: 2263-2275, 2008.

14. Kuthan H, Tsuji H, Graf $\mathrm{H}$ and Ullrich V: Generation of superoxide anion as a source of hydrogen peroxide in a reconstituted monooxygenase system. FEBS Lett 91: 343-345, 1978.

15. Tripathy D, Mohanty P, Dhindsa S, Syed T, Ghanim H, Aljada A and Dandona P: Elevation of free fatty acids induces inflammation and impairs vascular reactivity in healthy subjects. Diabetes 52: 2882-2887, 2003.

16. Lugrin J, Rosenblatt-Velin N, Parapanov R and Liaudet L: The role of oxidative stress during inflammatory processes. Biol Chem 395: 203-230, 2014.

17. Zeng MD, Fan JG, Lu LG, Li YM, Chen CW, Wang BY and Mao YM; Chinese National Consensus Workshop on Nonalcoholic Fatty Liver Disease: Guidelines for the diagnosis and treatment of nonalcoholic fatty liver diseases. J Dig Dis 9: 108-112, 2008

18. Ricchi M, Odoardi MR, Carulli L, Anzivino C, Ballestri S, Pinetti A, Fantoni LI, Marra F, Bertolotti M, Banni S, et al: Differential effect of oleic and palmitic acid on lipid accumulation and apoptosis in cultured hepatocytes. J Gastroenterol Hepatol 24: 830-840, 2009.

19. Livak KJ and Schmittgen TD: Analysis of relative gene expression data using real-time quantitative PCR and the 2(-Delta Delta C(T)) method. Methods 25: 402-408, 2001.
20. WHO Expert Consultation: Appropriate body-mass index for Asian populations and its implications for policy and intervention strategies. Lancet 363: 157-163, 2004.

21. Ip E, Farrell GC, Robertson G, Hall P, Kirsch R and Leclercq I: Central role of PPAR $\alpha$-dependent hepatic lipid turnover in dietary steatohepatitis in mice. Hepatology 38: 123-132, 2003.

22. Zhang X, Li S, Zhou Y, Su W, Ruan X, Wang B, Zheng F, Warner M, Gustafsson JA and Guan Y: Ablation of cytochrome P450 omega-hydroxylase 4A14 gene attenuates hepatic steatosis and fibrosis. Proc Natl Acad Sci USA 114: 3181-3185, 2017.

23. Garcia MC, Amankwa-Sakyi M and Flynn TJ: Cellular glutathione in fatty liver in vitro models. Toxicol In Vitro 25: 1501-1506, 2011.

24. Eid AA, Gorin Y, Fagg BM, Maalouf R, Barnes JL, Block K and Abboud HE: Mechanisms of podocyte injury in diabetes: Role of cytochrome P450 and NADPH oxidases. Diabetes 58: 1201-1211, 2009.

25. Sozen E and Ozer NK: Impact of high cholesterol and endoplasmic reticulum stress on metabolic diseases: An updated mini-review. Redox Biol 12: 456-461, 2017.

26. Duwaerts CC and Maher JJ: Mechanisms of liver injury in non-alcoholic steatohepatitis. Curr Hepatol Rep 13: 119-129, 2014.

27. Zangar RC, Davydov DR and Verma S: Mechanisms that regulate production of reactive oxygen species by cytochrome P450. Toxicol Appl Pharmacol 199: 316-331, 2004.

28. Hsu MH, Savas U, Griffin KJ and Johnson EF: Human cytochrome p450 family 4 enzymes: Function, genetic variation and regulation. Drug Metab Rev 39: 515-538, 2007.

29. Chavez-Tapia NC, Rosso N and Tiribelli C: In vitro models for the study of nonalcoholic fatty liver disease. Curr Med Chem 18: 1079-1084, 2011

30. Kang H and Koppula S: Houttuynia cordata alleviates high-fat diet-induced non-alcoholic fatty liver in experimental rats. Pharm Biol 53: 414-422, 2015.

31. Vornoli A, Pozzo L, Della Croce CM, Gervasi PG and Longo V: Drug metabolism enzymes in a steatotic model of rat treated with a high fat diet and a low dose of streptozotocin. Food Chem Toxicol 70: 54-60, 2014.

32. Kohjima M, Enjoji M, Higuchi N, Kato M, Kotoh K, Yoshimoto T, Fujino T, Yada M, Yada R, Harada N, et al: Re-Evaluation of fatty acid metabolism-related gene expression in nonalcoholic fatty liver disease. Int J Mol Med 20: 351-358, 2007.

33. Zhou Y, Luo P, Chang HH, Huang H, Yang T, Dong Z, Wang CY and Wang MH: Colfibrate attenuates blood pressure and sodium retention in DOCA-salt hypertension. Kidney Int 74: 1040-1048, 2008.

34. Miyata N, Taniguchi K, Seki T, Ishimoto T, Sato-Watanabe M, Yasuda Y, Doi M, Kametani S, Tomishima Y, Ueki T, et al: HET0016, a potent and selective inhibitor of 20-HETE synthesizing enzyme. Br J Pharmacol 133: 325-329, 2001.

35. Pessayre D: Role of mitochondria in non-alcoholic fatty liver disease. J Gastroenterol Hepatol 22 (Suppl 1): S20-S27, 2007.

36. Byrne CD: Fatty liver: Role of inflammation and fatty acid nutrition. Prostaglandins Leukot Essent Fatty Acids 82: 265-271, 2010.

37. Feldstein AE, Werneburg NW, Canbay A, Guicciardi ME, Bronk SF, Rydzewski R, Burgart LJ and Gores GJ: Free fatty acids promote hepatic lipotoxicity by stimulating TNF-alpha expression via a lysosomal pathway. Hepatology 40: 185-194, 2004.

38. Rolo AP, Teodoro JS and Palmeira CM: Role of oxidative stress in the pathogenesis of nonalcoholic steatohepatitis. Free Radic Biol Med 52: 59-69, 2012

39. Cnop M, Foufelle F and Velloso LA: Endoplasmic reticulum stress, obesity and diabetes. Trends Mol Med 18: 59-68, 2012.

40. Hui JM, Hodge A, Farrell GC, Kench JG, Kriketos A and George J: Beyond insulin resistance in NASH: TNF-alpha or adiponectin? Hepatology 40: 46-54, 2004

41. Morgan MJ and Liu ZG: Crosstalk of reactive oxygen species and NF- $\kappa \mathrm{B}$ signaling. Cell Res 21: 103-115, 2011.

This work is licensed under a Creative Commons Attribution-NonCommercial-NoDerivatives 4.0 International (CC BY-NC-ND 4.0) License. 